\title{
rapport annuel 1984 \\ des activités de recherches en France en Mécanique des Roches \\ (COMITÉ FRANÇAIS DE MÉCANIQUE DES ROCHES
}

\author{
Texte coordonné par P. BEREST \\ Directeur de Recherche \\ Laboratoire de Mécanique des Solides \\ Ecole Polytechnique*
}

\section{Résumé}

Les recherches effectuées en France dans le domaine de la Mécanique des Roches sont présentées ainsi que les centres de recherches où elles sont menées.

\section{Abstract \\ The main research carried out in France in the field of Rock Mechanics is indicated as well as the Research Centers where they are done.}

Avertissement:

Le présent rapport d'activité a été établi à partir d'une enquête réalisée par le Comité Français de Mécanique des Roches auprès de l'ensemble de ses membres. Des réponses individuelles ou collectives ont été reçues des organismes suivants:

- Bureau de Recherches Géologiques et Minières

- Centre d'Etude Technique de l'Equipement, Clermont-Ferrand

- Laboratoire Central des Ponts et Chaussées

- Ecole Nationale Supérieure des Techniques Industrielles et des Mines d'Alès

Laboratoire de Mécanique des Terrains et C.E.R.C.H.A.R.

(E.N.S.T.I.M., Alès)

Société Nationale Elf Aquitaine Production

- Institut de Physique du Globe

(Ecole des Mines de Nancy)

Laboratoire de Mécanique des Solides, Ecole Polytechnique

- Laboratoire de Génie Civil, Université de Montpellier II

C.E.R.C.H.A.R., Nancy (S.N.E.A.P.) (I.P.G.) (L.M.S. -X)

Centre d'Etude de Mécanique des Roches, Ecole des Mines de Paris

(C.E.M.R., Mines Paris) 


\section{GÉNÉRALITÉS}

L'activité de la Mécanique des Roches française paraît, bien normalement, refléter les tendances des domaines d'activité industriels auxquels elle concourt.

Ainsi, l'activité minière (incluant les carrières souterraines) ressent les effets d'une récession à l'échelle mondiale. Néanmoins, IE.N.S.T.I.M. d'Alès (stabilité, abattage) profite d'un relatif dynamisme minier régional; le C.E.R.C.H.A.R. (stabilité, soutènement, phénomènes dynamiques) s'appuie sur les besoins, qui demeurent importants, de l'industrie charbonnière nationale; pour le C.E.M.R.-Mines de Paris (stabilité, abattage, affaissements) l'activité minière demeure le thème d'études principales, en raison de solides traditions; malgré un contexte morose, le B.R.G.M. (stabilité, exhaure) voit augmenter ses interventions, en particulier à l'étranger. Il est néanmoins révélateur que la stabilité des ouvrages abandonnés suscite une part non négligeable de l'activité (C.E.R.C.H.A.R. et B.R.G.M.).

Cette tendance défavorable est encore plus nette dans d'autres secteurs traditionnels d'intervention de la Mécanique des Roches: les tunnels ne sont cités que par l'Ecole Polytechnique (problèmes de gonflement) et le C.E.M.R.-Mines de Paris (soutènement par cintre). Les barrages ne sont cités dans aucune réponse. Les stockages souterrains d'hydrocarbures sont un peu mieux traités (Ecole Polytechnique, C.E.M.R.-Mines de Paris, plus accessoirement S.N.E.A.P.) mais dans ce domaine également l'activité paraît réduite par rapport au niveau atteint pendant la décennie 1970-1980.

Les problèmes liés à l'exploration et à la production pétrolières sont, par contre, fortement représentés par S.N.E.A.P., avec une importante activité à l'étranger. Cette tendance est renforcée par l'utilisation des techniques pétrolières traditionnelles (dont la fracturation hydraulique) dans le développement d'utilisations nouvelles des ressources du sous-sol (géothermie sèche, gazéification in situ du charbon et des schistes bitumineux). Le dynamisme de ces nouvelles activités est souligné par l'intervention de nombreux organismes (I.P.G., B.R.G.M., Ecole Polytechnique, L.G.C. Montpellier...).

Elles ouvrent la possibilité de développements des recherches dans le domaine du couplage entre les écoulements de fluide, le comportement mécanique de la matrice rocheuse et les phénomènes thermiques. Ces thèmes sont également fortement présents dans le problème de l'enfouissement des déchets nucléaires, cité par le B.R.G.M., l'Ecole Polytechnique, le C.E.M.R.-Mines de Paris; ce problème paraît en voie d'occuper, comme dans de nombreux autres pays, une place très importante dans la Mécanique des Roches française.

Les études liées à la sécurité civile, déjà évoquées à propos des ouvrages miniers abandonnés, confirment leur importance avec les nombreux travaux liés à la sismicité et aux glissements de terrain (B.R.G.M.); au volcanisme et à la sismogénèse (I.P.G.)
Ainsi, aux fortunes diverses des activités plus traditionnelles s'oppose un développement assez dynamique associé à des techniques nouvelles: les activités de Génie Civil, d'extraction de minerai et d'exploitation conventionnelle des ressources énergétiques (à l'exception du domaine pétrolier) marquent fortement le pas alors que les recherches liées aux formes nouvelles de production d'énergie, à l'enfouissement des déchets et à la sécurité vis-â-vis des risques naturels connaissent un réel essor. Ces tendances paraissent, grosso modo, analogues à celles observées dans les autres pays.

Soixante-dix-neuf publications (thèses, articles, rapports) ont été citées dans les réponses à l'enquête; cinquante-neuf d'entre elles sont rédigées en langue française, vingt en langue anglaise.

\section{PROPRIÉTÉS DES ROCHES ET TECHNIQUES DE MESURE EN LABORATOIRE}

Le sel gemme a fait l'objet d'études de laboratoires par plusieurs équipes. Le C.E.M.R. (E.N.S.M.P.) dans l'optique du stockage souterrain d'hydrocarbures, a examiné le comportement rhéologique du sel à température inférieure à $80^{\circ} \mathrm{C}$. L'accent est porté sur le fluage sous contrainte triaxiale et l'essai D.R.B.I., mis au point au C.E.M.R. depuis plusieurs années. Le L.M.S. (Ecole Polytechnique) a effectué des essais de fluage de plusieurs mois sur du sel porté à différentes températures, jusqu'à $200^{\circ} \mathrm{C}$, pour les problèmes d'enfouissement de déchets nucléaires.

Dans le même laboratoire, le charbon a fait l'objet de nombreux essais en température, dans la perspective de la gazéification souterraine. Les essais triaxiaux à température élevée soulèvent un problèrne, compte tenu de la combustion spontanée du charbon. Cette difficulté a été partiellement levée par des essais de poinçonnement d'éprouvettes placées dans du plomb fondu. Le charbon et le grès ont fait l'objet d'essais au L.C.P.C., sous diverses sollicitations, pendant lesquels sont enregistrés et analysés le signal acoustique, qui peut être mis en rapport avec l'initiation et la propagation de fissures. On a montré que ce signal permet de distinguer les diverses phases d'évolution de la fissuration; on s'efforce d'utiliser ces résultats à l'interprétation de mesures acoustiques in situ.

L'I.P.G. a étudié les variations d'aimantation induites par les variations de l'état de contrainte appliqué à une roche. Une relation linéaire entre état de contrainte et susceptibilité magnétique a été mise en évidence sur une andésite et un basalte. Une étude numérique des effets d'un relâchement de contrainte, associée à un séisme sur l'aimantation induite du fait du piézomagnétisme, a été menée.

L'étude du comportement après la rupture des roches minières, sous faible confinement, a été poursuivie au C.E.M.R. (E.N.S.M.P.) en perfectionnant l'appareillage utilisé. Sur le même sujet, l'E.N.S.T.I.M. (Alès) a proposé un modèle théorique simple, fondé sur la théorie de l'endommagement, permettant de mettre en 
évidence des comportements type en compression monoaxiale.

Le granite a suscité des études motivées par le problème d'enfouissement des déchets nucléaires. Le C.E.M.R. (E.N.S.M.P.) a proposé, à partir d'études expérimentales du comportement thermomécanique de granites fissurés, une modélisation numérique du comportement d'un massif granitique. Le B.R.G.M. a étudié l'influence de la rugosité des épontes sur le comportement des fractures. Le L.M.S. (Ecole Polytechnique) a mis en évidence l'influence d'un chargement thermique sur l'état de fissuration.

\section{MOYENS DE CALCUL}

Le comportement des terrains élastoplastiques avec radoucissement a fait l'objet de codes de calcul, appliqués aux problèmes miniers, au C.E.M.R. (E.N.S.M.P.) et au C.E.R.C.H.A.R. Utilisé pour simuler le comportement des exploitations par chambres et piliers, ce comportement permet de mettre en évidence des instabilités globales (E.N.S.T.I.M., Alès). Les problèmes viscoplastiques ont donné lieu au développement du code V.I.P.L.E.F, au C.E.M.R. (E.N.S.M.P.) et à la création du code A.S.T.R.E.A., au L.M.S. Ecole Polytechnique. Ce dernier code accorde une grande importance aux phénomènes thermiques et a été conçu en vue du problème de l'enfouissement des déchets nucléaires dans le sel gemme. Un modèle couplé hydrothermomécanique des écoulements transitoires dans le milieu continu équivalent à une milieu fracturé à deux dimensions a été mis au point par le B.R.G.M. qui a également utilisé la géostatistique pour l'analyse de la densité de fracturation des massifs.

Le L.M.S. (Ecole Polytechnique) a proposé une solution viscoplastique explicite du problème de l'interaction entre terrains et soutènements dans une galerie circulaire qui généralise la méthode de convergenceconfinement.

\section{GÉOLOGIE, HYDROGÉOLOGIE, TECHNIQUES D'INVESTIGATION IN SITU, AUSCULTATION}

\section{Géologie}

La plupart des travaux réalisés dans ce domaine l'ont été au B.R.G.M. et pour certains d'entre eux à l'Ecole des Mines de Paris (Centre d'Information Géologique) et concernent la description, la quantification, l'analyse et la prévision de la fracturation naturelle affectant les massifs rocheux.

Beaucoup de ces travaux ont été effectués dans le cadre de programmes de recherche liés au problème du stockage dans le sous-sol des déchets radioactifs de haute activité et intéressent à ce titre plus particulièrement le granite, matériau dont l'étude a été faite par le
Royaume-Uni et la France dans le cadre de la Communauté Européenne.

C'est ainsi que des quantités très considérables de données sur la apetite fracturation" (celle que l'on peut observer à l'échelle d'un affleurement ou d'un front de taille) ont êté recueillies dans la mine d'uranium de Fanay-Augères, exploitée par la Cogema et située dans le granite de Saint-Sylvestre, en HauteVienne. Ces données, qui concernent tous les paramètres permettant de caractériser la fracturation (directions et pendages, dimensions, distance entre fractures, ouverture, morphologie, nature géologique de la discontinuité, remplissage, relations avec les autres discontinuités, etc.) ont servi et continuent de servir de support à de nombreuses études: structurales (reconstitution de l'histoire tectonique du massif, prévision de la fracturation en profondeur, évaluation des directions actuelles des contraintes in situ), hydrogéologiques (cf. paragraphe suivant) et statistiques.

Dans ce dernier domaine, des résultats prometteurs ont été obtenus avec l'application à la prévision de la densité de fracturation du massif des techniques issues de la géostatique.

En dehors de ce thème «fracturation», il convient de mentionner une approche très fine de l'influence des variations de faciès de calcaires très tendres (calcaires stampiens de l'Entre-Deux-Mers, en Gironde) sur leur comportement mécanique et leur altérabilité dans les anciennes carrières souterraines de pierre de taille, désormais abandonnées et menaçant ruine.

\subsection{Hydrogéologie}

L'essentiel des travaux réalisés dans ce domaine, à I'Ecole des Mines de Paris (Centre d'Informatique Géologique) et au B.R.G.M., concerne la caractérisation et la simulation des écoulements dans les milieux rocheux fracturés. Une voie particulièrement prometteuse s'est ouverte avec le début d'application à ces milieux du modèle de percolation introduit en 1957 par les mathématiciens britanniques BROABENT et HAMMERSLEY et développé en France par E. GUYON.

A noter par ailleurs la mise au point, à deux dimensions, de modẻles de calcul «hydrothermomécaniques» en milieu fracturé, aussi bien dans l'hypothèse du "milieu continu équivalent " que dans celle où les fractures sont traitées en tant que telles.

Les circulations de fluide dans le sous-sol font évidemment l'objet de recherches permanentes de la part de l'industrie pétrolière et, parmi celles-ci, il faut mentionner en particulier les travaux menés par la S.N.E.A.P. sur l'appréciation de l'anisotropie de perméabilité d'un milieu fissuré, liée en particulier à lanisotropie des contraintes in situ, et leurs applications à l'optimisation de la position de drains inclinés ou horizontaux.

\subsection{Techniques d'investigation in situ}

Les travaux réalisés et les résultats obtenus dans ce domaine sont nombreux et variés. 
Bien qu'il ne s'agisse pas à proprement parler d'investigation in situ, on peut mentionner, pour commencer, une première approche visant à utiliser la télédétection, et plus particulièrement les images qui seront fournies par le futur satellite d'observation terrestre Spot pour l'identification et le suivi de zones instables.

La mesure de contraintes in situ par fracturation hydraulique en sondage a fait l'objet de nombreux travaux, de la part de l'I.P.G. de Paris et du B.R.G.M. Des valeurs de contraintes ont ainsi pu être mesurées sur quatre sites, dans le Haut-Rhin, l'Allier et la Haute-Vienne, jusqu'à des profondeurs de l'ordre de $1000 \mathrm{~m}$. Il a été vérifié, à l'occasion de ces essais, que louverture de fractures dans différentes directions permettait une détermination du tenseur des contraintes plus complète que celle qu'autorise la fracturation hydraulique classique. Cette * orientation" des fractures peut se faire soit en modifiant la distribution des contraintes au voisinage du trou à l'aide de vérins, soit en tirant parti de la fracturation naturelle.

La connaissance de cette dernière est, dans tous les cas, fondamentale. Aux différents dispositifs déjà existants pour le relevé des orientations de fractures recoupant un sondage, est venu s'ajouter un nouvel appareil de prise d'empreinte mis au point par le Laboratoire régional des Ponts et Chaussëes de Clermont-Ferrand.

La mesure des contraintes n'est évidemment pas la seule application de la fracturation hydraulique, qui peut être utilisée pour accroître la perméabilité d'un massif, la plupart du temps par stimulation de fractures pré-existantes. Les essais réalisés par l'I.P.G. de Paris dans le granite du Mayet-de-Montagne (Allier) ont montré que l'anisotropie et l'hétérogénéité de résistance du massif rocheux pouvaient jouer un rôle aussi important que l'état dé contrainte naturel sur la direction des fractures créées ou stimulées par fracturation hydraulique, et ceci d'autant plus que le fluide est moins visqueux et que la vitesse d'écoulement est faible lors de l'amorce de celui-ci. Dans bien des cas, la détermination de la direction des fractures créées ou stimulées revêt une grande importance.

Au premier rang des techniques envisagées pour assurer cette détermination, figurent l'observation et l'interprétation de l'émission acoustique engendrée par les déformations du massif associées aux variations de pression interstitielle.

Cette technique a fait l'objet d'études de la part de la S.N.E.A.P. (en liaison avec lI.F.P. et l'A.R.T.E.P.) et de I.I.P.G. de Paris. Pour ce dernier, les applications visées ne se situaient pas exclusivement dans le contexte de la fracturation hydraulique (applications au domaine pétrolier et à celui des aroches massives haute température» ou de la gazéification in situ du charbon), mais également dans celui de la prévision des éruptions volcaniques et de l'étude des déformations liées à l'activité sismique.

En matière de mesure des contraintes in situ, il faut mentionner, pour terminer, les recherches entreprises par l'L.P.G. de Paris sur la relation entre variations de la susceptibilité magnétique d'une roche et variations de contrainte. Cette propriété a permis de reconstituer l'état de contrainte en profondeur à partir de mesures réalisées sur des échantillons prélevés en sondage à différentes cotes.

La contribution de l'industrie pétrolière ne se limite pas à l'émission acoustique, mentionnée ci-avant, et à laquelle on peut rattacher l'évaluation de l'état d'un puits par analyse des formes complètes des trains d'ondes transmis par la roche que permet de déterminer la sonde EVA, mise au point par la S.N.E.A.P.

Cette société s'est en effet intéressée, en outre, à une meilleure détermination des caractéristiques pétrophysiques des roches réservoirs, consolidées ou non, en vue d'une évaluation plus précise des récupérations primaires, et à une obtention et une interprétation plus valables d'enregistrements de paramètres (pression de fond) en liaison, pour ce dernier point, avec l'A.R.T.E.P. et avec la commission ad hoc de la S.I.M.R. dont l'animateur pour l'Europe est F.-H. CORNET.

En matière d'investigation in situ, il faut mentionner, pour terminer, une approche originale, développée par le Laboratoire de Mécanique des Solides (Ecole Polytechnique) du problème de l'évaluation du volume d'une cavité souterraine (en particulier dans le cas où celle-ci a été créée par dissolution du sel). La méthode utilisée repose sur une analyse des oscillations dans le forage d'accès à la cavité.

\subsection{Auscultation}

Une part importante des travaux réalisés en matière d'auscultation concerne le domaine minier, dans lequel le C.E.R.C.H.A.R. poursuit une tâche permanente d'amélioration des soutènements en voie ou en taille, reposant entre autres sur l'acquisition et l'analyse de nombreuses mesures in situ (convergence, expansion dans les terrains, déformations des éléments de soutènement, tous ces paramètres étant enregistrés in situ sur cassettes).

La prévision des phénomènes dynamiques, ruptures soudaines et violentes du massif en cours d'exploitation, comme il s'en produit aux Houillères de Provence, a fait l'objet de la mise au point d'un dispositif combinant l'utilisation de trous tests et celle de l'écoute sismo-acoustique.

En matière d'émission acoustique, le passage de l'échelle des essais de laboratoire à celle du terrain a continué de faire lobjet, au L.C.P.C., de recherches qui ont permis de corréler l'activité acoustique avec le diagramme effort-déformation.

Avant de clore le paragraphe consacré à l'ausculation dans le domaine minier, il convient de mentionner l'équipement en extensomètres et plots de mesure de convergence d'un quartier de la mine d'or de Salsigne (Aude) destiné à être exploité par sous-niveaux abattus. Cette opération vise à permettre par comparaison des résultats fournis par les moyens de calcul classiquement mis en ceuvre avec le comportement réel des chantiers. 
Dans le domaine des travaux souterrains, depuis 1983. le B.R.G.M., auquel s'est associé le L.M.S., a participé aux travaux de recherche réalisés par le Centre d'Etudes Nucléaires de Belgique dans le laboratoire souterrain de Mol. L'objectif de ces travaux est d'étudier in situ, et en vraie grandeur, le comportement d'une argile profonde.

Dans le cadre de son activité en sismologie et volcanologie, l'I.P.G. de Paris a été amené à adapter des systèmes très précis de mesure des déformations et des inclinaisons du sol et du sous-sol en vue de leur installation dans des zones sismiques (Arette, PyrénéesAtlantiques) et volcaniques (Guadeloupe, Martinique, Réunion, Etna), mais également sur des sites où ont été réalisées des opérations de fracturation hydraulique. Les appareils utilisés se sont montrés capables d'enregistrer la propagation de fractures horizontales à quelques centaines de mètres de profondeur.

\section{EXCAVATIONS SOUTERRAINES}

Les mines, comme indiqué dans l'introduction, ont motivé une part importante des recherches du C.E.M.R. (E.N.S.M.P.), du C.E.R.C.H.A.R., du B.R.G.M., de l'E.N.S.T.I.M. (Alès).

Un important effort de réflexion sur la modélisation a accompagné la collecte de données relatives à des mesures au fond au C.E.R.C.H.A.R. (voir paragraphe: Techniques de renforcement). Les phénomènes dynamiques (ruptures soudaines et violentes du massif en cours d'exploitation) qui font courir des risques graves au personnel, et portent un préjudice économique considérable, exigent une compréhension affinée de leurs mécanismes. L'influence de la profondeur et de la tectonique a été envisagée, à travers la mesure des contraintes in situ (vérin plat et surcarottage). La prévision s'oriente vers la surveillance par trous test et écoute sismo-acoustique (Houillères de Provence) ou analyse du disquage dans des sondages de reconnaissance (Houillères de Provence)

Le B.R.G.M., à travers des projets miniers en France et à l'étranger, a pu confirmer son expérience en matière d'analyse de stabilité et d'exhaure minière.

Le C.E.M.R. (E.N.S.M.P.) a porté un effort particulier sur les méthodes d'exploitation, par foudroyage, et la mesure des affaissements de surface (en Lorraine). Les phénomènes d'angle d'influence ont pu être précisés; une méthode de prévision des coups de charge a été proposée.

L'E.N.S.T.I.M. (Alès) a étudié la stabilité de divers types de travaux miniers.

- Les carrières souterraines à faible profondeur, le plus souvent abandonnées, ont également fait l'objet d'études importantes du B.R.G.M. (altérabilité des calcaires du Bordelais, confortement des piliers abandonnés). Le C.E.R.C.H.A.R. a plus particulièrement approfondi le cas des exploitations où la roche est en état de post-rupture; l'analyse des mécanismes et des causes des ruptures a contribué à la mise au point d'un diagnostic de stabilité.

- Les stockages souterrains d'hydrocarbures, et plus particulièrement les cavités en gaz naturel réalisées dans le sel par Gaz de France, sont étudiés par le C.E.M.R. (E.N.S.M.P.) qui a affiné le logiciel V.I.P.L.E.F, et par le L.M.S. (Ecole Polytechnique) qui propose de porter l'attention sur la dissymétrie de l'écoulement plastique da aux forces de gravité. Le L.M.S. (Ecole Polytechnique) a proposé une analyse d'accidents variés ayant affecté les stockages souterrains.

- La gazéification en place du charbon a donné lieu, à l'initiative du G.E.G.S. (quí regroupe G.D.F., C.E.R.C.H.A.R., I.F.P et B.R.G.M.), à la réalisation d'une liaison par fracturation hydraulique à la base d'un doublet de forage à 1000 mètres de profondeur (Haute Deule). Le B.R.G.M. et l'I.P.G. ont été associés au premier essai européen de gazéification en place de schistes bitumineux mené par la C.F.P., à Tranqueville (Vosges).

- La géothermie a fourni â divers organismes matière à des recherches originales. Le projet "Géothermie Profonde», qui associe l'I.N.A.G., l'A.F.M.E. et le B.R.G.M., envisage l'étude en vraie grandeur de la possibilité d'exploiter la chaleur des roches profondes peu perméables.

L'I.P.G., associé à ce programme, a obtenu des résultats importants en matière de fracturation hydraulique. On admettait communément qu'une fracture hydraulique se développe perpendiculairement à la direction de la contrainte principale mineure. Des essais dans le granite de Mayet-de-Montagne, ont permis de montrer que l'anisotrope et l'hétérogénéité de résistance du massif jouent un rôle d'importance au moins comparable à celui de l'état de contraintes sur la géométrie des fractures développées. Par ailleurs, la mesure des contraintes en place au moyen de la fracture hydraulique a fait lobjet de nouveaux développements: l'utilisation de plans de faiblesse préexistants, ou la modification de l'état de contraintes au fond par des vérins hydrauliques, fournissent un ensemble de données plus riches et plus précises que la fracturation hydraulique classique (voir aussi le paragraphe: "Reconnaissances in situ $)$. Le L.G.C. (Montpellier) a également mené sur ce thème des études, dont on trouvera l'écho dans d'autres paragraphes. Le B.R.G.M. a été informé du projet anglais de Cornouailles.

- L'enfouissement des déchets nucléaires suscite un nombre croissant d'études. Sur le thème du granite, pour lequel les études ont commencé depuis plusieurs années déjà, le B.R.G.M. a développé, à la demande du C.E.A. (I.P.S.N.), d'importantes recherches sur l'analyse de la fracturation naturelle des massifs; le C.E.M.R. (E.N.S.M.P.) a développé un logiciel adapté au calcul de l'évolution thermomécanique d'un massif fracturé. Ces points sont développés par ailleurs.

- L'argile a donné lieu à une participation du B.R.G.M. aux travaux du laboratoire souterrain de Mol, en Belgique. Le B.R.G.M. et le L.M.S. (Ecole Poly- 
technique) ont mené conjointement une campagne d'essais mécaniques sur des argiles françaises. Le développement d'un logiciel adapté au B.R.G.M. est évoqué par ailleurs.

- De même, l'apparition récente du thème «sel gemme a conduit le L.M.S. (Ecole Polytechnique), à la demande de l'A.N.D.R.A., à créer les moyens d'essais mécaniques (fluage de longue durée) adaptés et à développer un logiciel de calcul élastothermoviscoplastique, évoqué dans d'autres rubriques.

\section{OUVRAGES DE SURFACE}

Les travaux de recherche développés en 1983 dans le domaine des ouvrages de surface s'articulent autour de trois axes principaux:

- La modélisation des massifs rocheux superficiels;

- La prise en compte des risques géologiques (éboulements rocheux, risques sismiques);

- Le développement des méthodes d'auscultation et de surveillance.

\subsection{Modélisation des massifs rocheux superficiels}

Le rôle essentiel joué par les discontinuités dans les propriétés et le comportement des massifs rocheux superficiels conduit naturellement à développer les recherches dans ce domaine pour l'étude des ouvrages de surface (barrages, fondations, falaises et versants rocheux).

L'amélioration des méthodes de traitement des données structurales des massifs rocheux par une meilleure prise en compte des paramètres descriptifs, des plans de discontinuité (localisation, nature, orientation, espacement, extension, remplissage,...) a fait l'objet d'un travail de mise au point important dans plusieurs laboratoires (B.R.G.M., L.P.C.). Des programmes de traitement informatique des données structurales permettant également l'analyse et la visualisation de ces données ainsi que l'édition et le dessin automatique ont été développés. Les possibilités du traitement informatique ont permis de développer une approche géostatistique de la densité de fracturation et une application intéressante à l'analyse d'un site granitique (B.R.G.M.).

La représentation tridimensionnelle de la structure et de la géométrie des massifs superficiels au moyen de blocs-diagrammes permettant la visualisation dans l'espace d'un modèle du massif comportant la topographie, la structure, le tracé de l'ouvrage projeté a été développée par le L.C.P.C.

Les programmes informatiques mis au point permettent un traitement interactif des données géométriques et structurales sur une console de visualisation et l'édition de dessin automatique.
L'étude du comportement à la rupture et post-rupture des discontinuités dans les massifs rocheux constitue un axe de recherche important. Sur le plan expérimental, l'étude du comportement au cisaillement des discontinuités dans les roches et l'analyse de l'influence de la géométrie des épontes a été développée sur des roches ou des matériaux artificiels (L.P.C., B.R.G.M.). L'analyse du développement du processus de cisaillement dans les plans de discontinuité dans les roches a été plus particulièrement étudié par le L.P.C. dans le cadre de recherches sur les mécanismes de développement de la rupture dans les massifs superficiels.

Dans le domaine de la modélisation, un travail de recherche a été développé sur les modèles numériques d'analyse du comportement à la rupture et postrupture des discontinuités dans les roches, permettant de traduire les phénomènes de dilatance et de broyage qui se développent pendant le cisaillement (L.P.C.).

\subsection{Prise en compte des risques géologiques}

Les recherches développées dans plusieurs laboratoires dans le domaine du microzonage sismique ont conduit à entreprendre des études sur l'influence des données géologiques, géotechniques et topographiques sur la réponse dynamique des sites exposés aux sollicitations sismiques (B.R.G.M., L.P.C.) et sur les conséquences possibles quant à la stabilité des sols de fondation. Ces travaux ont été réalisés en coordination avec l'Association Française de Génie Parasismique.

Dans le domaine des risques liés aux éboulements rocheux, des recherches ont été développées sur l'analyse des mécanismes de propagation des éboulements. Ce thème a fait l'objet de recherches sur la modélisation des phénomènes de propagation (L.P.C., B.R.G.M.). Des modèles numériques bidimensionnels ou tridimensionnels ont été développés, et permettent d'analyser, en fonction des données topographiques, des paramètres géomécaniques, des caractéristiques de l'éboulement et des caractères spécifiques du site (obstacles naturels ou artificiels), les conditions de propagation des masses éboulées (trajectoires, extension, énergie).

L'étude des ouvrages de protection a fait l'objet de recherches dans le domaine des systèmes déformables à dissipation d'énergie (L.P.C.).

\subsection{Auscultation et surveillance}

L'analyse des déplacements et des déformations à l'auscultation et à la surveillance des ouvrages de surface a fait l'objet de recherches de la part de plusieurs laboratoires. Des mëthodes de photogrammétrie aérienne de précision ont été appliquées conjointement à des mesures géodésiques, à l'étude des déformations du volcan du Piton de la Fournaise à La Réunion (I.P.G., I.G.N.). A plus petite échelle, la mise en ceuvre de réseaux de mesure de métrologie dimensionnelle de précision permettant une auscultation fine des déformations d'un massif rocheux à l'échelle de l'hectare a été développée (L.P.C.). Dans le domaine des mesures de haute précision, les 
mesures extensométriques et clinométriques ont fait l'objet d'applications nouvelles à l'étude des déformations de massifs superficiels (I.P.G.).

L'auscultation et la surveillance des zones rocheuses, instables, en site de montagne ont conduit à développer des systèmes de télémesure et de télésurveillance spécifiques adaptés aux conditions d'accès difficiles et aux contraintes climatiques sévères (L.P.C.). Plusieurs sites importants ont été instrumentés dans les Alpes. Parallèlement, des études ont été entreprises sur les mécanismes de déformation qui affectent certaines zones instables suivies depuis plusieurs années en vue de mettre en évidence des modèles de comportement et des critères d'évolution (L.P.C.).

\section{COMMINUTION}

Dans ce domaine, on doit d'abord rappeler les travaux sur la forabilité en fonction des paramètres de forage (poids, rotation, boue, forme de l'outil, etc.), menés par S.N.E.A.P., en particulier au Laboratoire de Boussens où sont simulées les conditions très sévères des gisements.

Le C.E.M.R. (E.N.S.M.P.), en liaison avec le C.E.R.C.H.A.R., a étudié l'action des pics; un code de calcul prenant en compte le radoucissement a permis des simulations qui clarifient la notion d'effort de refoulement et permettent de prévoir les conséquences de l'usure des pics. L'étude expérimentale a été faite à échelle réduite, sur banc d'essais rotatif et linéaire.

Le L.G.C. (Montpellier) a développé des expériences de fracturation thermique pour le problème de la géothermie à grande profondeur en portant l'attention sur les roches anisotropes, éventuellement préfissurées, sollicitées par des chargements hydrauliques, dans le but de dégager des critères de rupture globaux.

L'E.N.S.T.I.M. (Alès) a participé à une étude sur les mécanismes de l'abattage à l'explosif; la cinématographie rapide permet de mettre en évidence les lois générales du mouvement durant l'abattage et la projection des fragments. Un modèle informatique de recomposition du tas de fragments abattus a été proposé.

\section{TECHNIQUES DE RENFORCEMENT DES MASSIFS ROCHEUX}

Le soutènement en voies, par cintres coulissants et le rôle du garnissage font l'objet d'une étude en cours au C.E.R.C.H.A.R., qui comporte une modélisation et des mesures in situ de convergence, de déformation des éléments de soutènement et d'expansion dans les terrains.

Des essais d'écrasement de cintres en vraie grandeur, menés par le C.E.M.R. (E.N.S.M.P.), en liaison avec la S.N.C.F., mettent en évidence l'importance du garnissage et des assemblables. Ils doivent conduire à des règles de dimensionnement utilisant une modélisation numérique.
L'étude du soutènement en taille est menée au C.E.R.C.H.A.R pour les conditions variées de terrains rencontrées dans les charbonnages (pendages très inclinés, couches très épaisses,...). Les mesures des efforts et des déplacements, dans le soutènement marchant et dans les terrains, ont suscité un important travail de dépouillement statistique correspondant aux données recueillies depuis une vingtaine d'années dans les houillères françaises.

Le confortement artificiel des piliers abandonnés des carrières souterraines du Bordelais a fait l'objet d'études du B.R.G.M. Le problème de la stabilité des pentes a conduit le même organisme à développer des essais de drainage de puits à drains rayonnants (falaises de Biarritz).

\section{SUJETS CONNEXES A LA MÉCANIQUE DES ROCHES}

\section{Thermodynamique des milieux polyphasiques ou complexes}

Ce thème est développé au L.G.C. (Montpellier) dans les domaines des sols et des milieux rocheux.

\section{- Domaine des milieux poreux et des sols}

La modélisation donne lieu à de nombreux développements pour diverses applications en relation avec les grands axes de recherche finalisée comme le stockage d'énergie dans le sol; des perspectives très encourageantes apparaissent pour le séchage, ainsi que pour des applications interdisciplinaires avec l'agronomie.

L'orientation de cette modélisation vers la compréhension de phénomènes plus fins, à l'aide de techniques d'homogénéisation et de macroscopisation est en très bonne voie.

\section{- Domaine des milieux rocheux homogènes, poreux ou fissurés}

La généralisation des études thermodynamiques entreprises pour les sols est commencée actuellement pour des roches à la fois poreuses et fissurées. Ces études ont été entreprises à la suite d'une demande du secteur industriel, pour évaluer les échanges de masse et d'énergie en géothermie à moyenne profondeur.

\section{Génie pétrolier}

Un programme très important d'études est mené par S.N.E.A.P. pour les problèmes de gisement, de forage et de production. Plus particulierement, on cherche à améliorer la définition mécanique du milieu dans les simulations de production, qui a été probablement très négligée jusqu'ici en comparaison de la modélisation de l'écoulement. L'analyse des phénomènes de rupture en forage a fait l'objet d'une typologie permettant l'identification des mécanismes fondamentaux impliqués. 
